\title{
MÉTODOS TERAPÊUTICOS INDICADOS NO TRATAMENTO DO CÂNCER DE MAMA GESTACIONAL
}

\section{THERAPEUTIC METHODS INDICATED IN THE TREATMENT OF GESTATIONAL BREAST CANCER}

\section{Amanda Batista Pedrosa;}

Bacharel em Enfermagem. Alfa - Faculdade de Almenara, Almenara, Minas Gerais, Brasil.

\section{Maria das Graças Gomes de Oliveira}

Bacharel em Enfermagem. Alfa - Faculdade de Almenara, Almenara, Brasil.

Viviane Amaral Toledo Coelho

Doutora. Autor parar correspondência;. Faculdade de Almenara, Almenara, Brasil.

E-mail: vivianeatc@yahoo.com.br

\section{Ednardo de Souza Nascimento}

Mestre. Alfa - Faculdade de Almenara, Almenara, Brasil.

\section{Creonice Santos Bigatello}

Especialista. Alfa - Faculdade de Almenara, Almenara, Brasil.

Recebido: 00/00/2018 - Aceito: 00/00/2018

\section{RESUMO}

O presente estudo tem como objetivo principal, descrever os métodos terapêuticos indicados no tratamento do câncer de mama gestacional, além de discutir a forma de condução do tratamento terapêutico neste tipo de patologia e as questões que ele pode vir a desencadear a partir do diagnóstico. Trata-se de um estudo bibliográfico, exploratório e descritivo, embasado em pesquisas publicados entre os anos 2000 a 2020. A neoplasia mamaria gestacional não engloba apenas o câncer diagnosticado durante o período de gravidez, mas envolve também aqueles que ocorrem até um 
ano após o parto. Esta patologia pode ser mais difícil de ser diagnosticada quando a mulher está grávida porque as alterações hormonais durante a gravidez fazem com que as mamas se tornem maiores, granuladas e/ou sensíveis, o que dificulta 0 diagnóstico. Cabe a equipe de enfermagem orientar os pacientes sobre uma alimentação saudável, e práticas de atividades físicas. Já que, segundo alguns estudos com esses hábitos é possível reduzir até $28 \%$ o risco de a mulher desenvolver neoplasia mamaria. A amamentação também é considerada um fator protetor contra esta patologia.

Palavras-chave: Neoplasia da Mama; Gestação; Complicações neoplásicas na Gravidez.

\section{ABSTRACT}

The present study has as main objective, to describe the therapeutic methods indicated in the treatment of gestational breast cancer, in addition to discussing the way of conducting therapeutic treatment in this type of pathology and the issues that it may trigger from the diagnosis. This is a bibliographic, exploratory and descriptive study, based on research published between the years 2000 to 2020. Gestational breast cancer does not only include cancer diagnosed during the period of pregnancy, but also involves those that occur up to one year after delivery. This pathology can be more difficult to be diagnosed when the woman is pregnant because hormonal changes during pregnancy cause the breasts to become larger, granular and / or sensitive, which makes diagnosis difficult. The nursing team is responsible for guiding patients on healthy eating and physical activity practices. Since, according to some studies with these habits, it is possible to reduce up to $28 \%$ the risk of women developing breast cancer. Breastfeeding is also considered a protective factor against this pathology.

Keywords: Breast Neoplasm; Gestation; Neoplastic complications in pregnancy.

\section{Introdução}

Câncer é o nome dado a um conjunto de mais de 100 doenças que têm em comum o crescimento desordenado de células, que invadem tecidos e órgãos. Dividindo-se rapidamente, estas células tendem a ser muito agressivas e incontroláveis, determinando a formação de tumores, que podem espalhar-se para outras regiões do corpo. O câncer representa $21 \%$ de todos os óbitos no mundo, esse impacto afeta principalmente os países de baixo e médio desenvolvimento, especialmente por mortes prematuras (PRADO et al., 2020).

O câncer de mama é uma doença causada pela multiplicação desordenada de células da mama. Esse processo gera células anormais que se multiplicam, formando um tumor. Há vários tipos de câncer de mama. Por isso, a doença pode evoluir de diferentes formas. Alguns tipos têm desenvolvimento rápido, enquanto outros crescem mais 
lentamente. Esses comportamentos distintos se devem a característica próprias de cada tumor. O câncer de mama também acomete homens, porém é raro, representando apenas 1\% do total de casos da doença. (INCA - Instituto Nacional do Câncer, 2020)

No Brasil, as estimativas de incidência de câncer de mama para o ano de 2020 são de 66.280 casos novos. Em 2017, foram 16.924 óbitos, sendo 16.724 mulheres e 203 homens (INCA, 2020). A neoplasia mamária é a segunda causa de câncer associada à gravidez, acontece em uma proporção de 1/3.000 a 1/10.000 nas gestantes entre 32 e 38 anos, ultrapassada apenas pelo câncer de colo de útero. De acordo com Rezende et al., (2005), o câncer de mama gestacional envolve não só o câncer diagnosticado na gravidez, mas pode manifestar-se após a concepção, no ano em que a mulher teve seu filho ou durante o período de amamentação. A expressividade da doença em período gestacional representa $0,2 \%$ a $3,8 \%$ de todos os cânceres.

Segundo Costa et al., (2006) o risco de desenvolver câncer de mama associado a gravidez está altamente ligado a idade materna: depois dos 30 anos de idade é a neoplasia mais frequente diagnosticada, a uma larga distância das outras. As mulheres com câncer de mama associado à gravidez tendem a ter o diagnóstico num estágio mais avançado e, são múltiplos fatores que contribuem para isso, o mais determinante então, parece ser a dificuldade de avaliar tumores mamários em mulheres grávidas devido ao ingurgitamento da glândula secundário às alterações hormonais características da gravidez. De acordo com Silva (2017) a prevalência deste tipo de câncer pode aumentar nos próximos anos em consequência da propensão da mulher em ter menos filhos e do adiamento do primeiro parto, determinados pelas mudanças nos hábitos de vida da mulher contemporânea.

O diagnóstico dessa neoplasia acarreta sofrimento às pacientes, gerando grandes conflitos emocionais na mulher, conforme afirmam Maluf et al., (2005), em especial quando ocorre na vigência de uma gravidez. O binômio mãe-feto não pode ser ignorado durante o manejo do câncer de mama associado à gestação, o que leva, muitas vezes, a conflitos de conduta e prognóstico. Diante de uma neoplasia mamaria durante a gestação, a escolha dos métodos terapêuticos indicados para o tratamento ideal, precisa ser baseado em estratégias que visem manter o equilíbrio entre o bem-estar da mãe e também do feto, minimizando os riscos para ele e promovendo o máximo de benefícios para ela (ASHWORTH, 2016).

De acordo com os estudos disponíveis na literatura atual, o tratamento irá depender do tamanho e localização do tumor, mas principalmente do tempo gestacional. Portanto, alguns métodos são contraindicados para essas mulheres, pois podem causar malefícios ao feto. Dentre estes procedimentos destacam-se a radioterapia, a hormonioterapia e a terapia molecular alvo. 
No cenário atual, ainda existe uma escassez de estudos capazes de evidenciar e garantir a segurança do uso de fármacos, principalmente os quimioterápicos no tratamento do câncer de mama gestacional, uma vez que esta patologia não é muito comum e também pouco discutida e divulgada na sociedade. Contudo, o fato das mulheres estarem optando por adiar a gestação para a terceira e até mesmo quarta década de vida, faz com que esta doença não seja tão rara assim, e levanta a urgência de mais discussões a respeito da neoplasia mamaria na gestação. (SILVA, 2017).

A forma de conduzir o tratamento terapêutico neste tipo de patologia e as questões que ele pode vir a desencadear a partir do diagnostico, serão abordadas neste estudo, a fim de despertar e esclarecer a sociedade quanto a necessidade de mais conhecimento nesta área, sobretudo diretrizes apropriadas que visem uma melhor condução para a resolução do problema.

Desse modo, este trabalho visa descrever os métodos terapêuticos indicados na condução do tratamento do câncer de mama gestacional, destacando os métodos preventivos existentes e os fatores de risco que podem desencadear a mesma.

\section{Revisão Bibliográfica}

\subsection{Caracterização do câncer}

O câncer, termo designado de forma genérica para representar mais de 200 doenças. Embora existam muitos tipos de câncer, todos começam devido ao crescimento e multiplicação anormal e descontrolado das células. A enfermidade também é conhecida como neoplasia (THULLER et al., 2003).

O câncer de mama é uma doença causada pela multiplicação desordenada de células da mama. Esse processo gera células anormais que se multiplicam, formando um tumor. Há vários tipos de câncer de mama. Por isso, a doença pode evoluir de diferentes formas. Alguns tipos têm desenvolvimento rápido, enquanto outros crescem mais lentamente. Esses comportamentos distintos se devem a característica próprias de cada tumor. O câncer de mama também acomete homens, porém é raro, representando apenas $1 \%$ do total de casos da doença. (INCA 2020).

Quando o câncer de mama é diagnosticado em uma mulher gravida é denominado câncer de mama gestacional ou câncer de mama associado a gravidez. O câncer de mama é diagnosticado em 1 em cada 3000 mulheres gravidas. Mas 0 câncer de mama é o tipo mais comum de câncer diagnosticado durante a gravidez, 
durante a amamentação ou no primeiro ano após o parto. Os cânceres de mama podem ser mais difíceis de serem diagnosticados quando a mulher está grávida. Alterações hormonais durante a gravidez fazem com que as mamas se tornem maiores, granuladas e/ou sensíveis (PRADO et al., 2020).

\subsection{Aspectos Epidemiológicos e contextuais}

Em nível mundial, o câncer (CA) de mama é o segundo mais frequente, perdendo somente para o de pulmão. No Brasil, é a neoplasia maligna que mais acomete as mulheres. Segundo o Ministério da Saúde, a neoplasia mamária perde apenas para as doenças cardiovasculares como a doença que mais mata, como consequência do diagnóstico tardio. Por conta dessa alta incidência, o câncer de mama é uma das doenças mais temidas pelas mulheres, seja por medo da morte, do tratamento ou da cirurgia de retirada da mama, que acaba sendo uma mutilação para a imagem corporal da mulher (CIPRIANO et al.,2015).

A estimativa de 2016 do Instituto Nacional de Câncer José Alencar Gomes da Silva (INCA) refere que os países subdesenvolvidos serão responsáveis por $80 \%$ dos mais de 20 milhões de casos novos de câncer estimados para 2025 (PANIS et al., 2018). Entre o aumento no número de casos de neoplasias, apresentam-se em destaque as ocorrências em mulheres em idade fértil, as quais denotam sérias consequências em seu futuro reprodutivo (PANIS et al., 2018) (MONTEIRO et al., 2013) (MONTEIRO et al., 2019). Dentre estas pacientes em idade fértil com diagnóstico de câncer, existe uma pequena, porém, relevante parcela de mulheres que tiveram diagnóstico de câncer durante a gestação ou que, no decorrer do tratamento/seguimento de uma neoplasia, engravidaram. A literatura evidencia que este número é crescente nas últimas décadas (MONTEIRO et al., 2019) (ANTONELLI et al., 1996).

Segundo Araújo e Centeno (2010); A maioria dos tumores mamários associados à gravidez (80-100\%) são carcinomas ductos invasivos. Já os tumores inflamatórios representam 1,5-4\%, apesar de, alguns estudos realizados com poucas amostras, que não apresentam resultados muito esclarecedores, apontarem para uma maior incidência deste tipo de neoplasia em mulheres grávidas. 0 
Ministério da saúde (2010); complementa que, a maioria dos estudos realizados apontam que a gravidez não é um fator de mau prognóstico, mas assinala que na mesma, possa se desenvolver de forma mais agressiva, embora os piores resultados que se observam, sejam relacionados diretamente ao atraso no diagnóstico e no tratamento, e não apenas às características biológicas dos tumores.

De acordo com Rezende et al. (2005), a neoplasia mamaria gestacional não engloba apenas o câncer diagnosticado durante o período de gravidez, mas envolve também aqueles que ocorrem até um ano após o parto. Mottola Jr et a., (2002); acrescenta que este tipo de patologia representa cerca de $0,2 \%$ a $3,8 \%$ de todos os tumores que podem ocorrer durante a gestação, tendo uma prevalência de um caso em cada 3.000, ou de um caso para 10.000 gestações. Ambos ressaltam que esta enfermidade manifesta-se como a segunda causa de neoplasia associada à gravidez, ultrapassada apenas pelo câncer de colo uterino.

Estima-se que o câncer complique cerca de 3.500 gestações nos Estados Unidos, com uma incidência de 1 para 1.000. Os processos malignos mais comuns durante a gravidez são: o câncer de colo do útero, câncer de mama, melanoma, câncer de ovário, câncer de tireoide, leucemia, linfoma e câncer colo retal. O câncer de mama representa $0,2 \%$ a $3,8 \%$ de todos os cânceres que ocorre na gestação. Ocorrem numa relação de 1/3.000 a 1/10.000, gestações na faixa etária de 32 a 38 anos, dependendo do país estudado, sendo que algumas séries atuais apresentam associação de 1/2.000 gestações. (GRENDYSJR. 2001, apud, COSTA et al.,2006).

Este tipo de neoplasia vem crescendo de forma gradativa, pois, as mulheres estão optando por adiar cada vez mais a gestação. Visto que, a partir das décadas de 1970 e 1980 surgiu o movimento feminista e, aos poucos, as mulheres foram conquistando maior independência tanto profissional quanto em relação à família e, dentro do modelo econômico introduzido atualmente, as mulheres conquistaram seu espaço na sociedade. Toda esta transformação feminina repercutiu também na medicina moderna, principalmente na obstetrícia, pois frequentemente as mulheres estão retardando a gravidez para a terceira e até mesmo quarta década de suas vidas. Como consequência as doenças que geralmente acometem mulheres em 
idade adulta, estão cada vez mais sendo associadas às gestações mais tardias (MOTTOLA JR, et al.,2002).

De acordo com Reed et al., (2003) a neoplasia mamária desenvolvida durante o período gestacional, quando mais cedo for descoberta melhor, pois este fator aumenta as chances de cura desta paciente, já que é possível optar por utilizar métodos terapêuticos mais indicados para a condução do tratamento. Ademais, o atraso no diagnóstico é muito prejudicial para a escolha de uma intervenção ideal em cada caso. O fato é que a neoplasia na gestação é muito difícil de ser diagnosticada, visto que as alterações fisiológicas que ocorrem na mama durante a gestação podem impedir e até mesmo atrapalhar o diagnóstico. Entretanto, o prognostico para esta mulher portadora de câncer de mama gestacional, é semelhante ao das portadoras de câncer de mama fora deste período, sendo a metástase para o linfonodo o principal fator prognostico em ambos os casos.

\subsection{Diagnóstico e patogenia do Câncer}

De acordo, com INCA o câncer de mama gestacional é caracterizado por um crescimento rápido e desordenado de células da mama, que adquirem a capacidade de se multiplicarem de forma acelerada. Estas tendem a ser muito agressivas e incontroláveis, provocando a formação de tumores malignos, que podem espalharse para outras partes do organismo. Pode ser percebido através de uma massa palpável, indolor, dura e irregular, presente na mama, mas há tumores que apresentam uma consistência branda, são globosos e bem definidos. Outros sinais de câncer de mama podem aparecer, como edema cutâneo, dor, inversão do mamilo, hiperemia, descamação ou ulceração do mamilo, e secreção papilar, podendo ser transparente, rosada ou avermelhada devido à presença de glóbulos vermelhos.

A ultrassonografia das mamas e a mamografia são indicadas sempre quando houver uma lesão suspeita na mama da gestante, associado aos sinais e sintomas explícitos anteriormente. Seguindo os mesmos critérios de uma pessoa diagnostica com câncer de mama, fora da gravidez, com exames de imagens e biopsia. Porém a 
gestante tem um tratamento diferenciado, pelo fato de apresentar alterações hormonais provenientes da própria gestação (INCA, 2017).

\subsection{Fatores de Risco da Doença}

Os fatores de risco para Câncer de mama gestacional ainda não são muito definidos, inúmeras razões estão relacionadas ao aumento do risco de desenvolver o problema, dentre eles: causas endócrinas, idade, fatores relacionados a hábitos de vida e fatores genético-hereditários. A gravidez é uma das três fases da vida da mulher em que ocorrem grandes modificações hormonais, são elas; o início da menarca, a primeira gestação e início da menopausa. Sendo a neoplasia mamaria constatada como uma patologia hormônio dependente, ou seja, aquela cujo seu crescimento e desenvolvimento acontecem pela ação de hormônios, em especial o estrógeno, foi percebido que a gestação pode ser uma condição com risco aumentado para este tipo de câncer. O uso de contraceptivos orais também é considerado um motivo de risco pela Organização Mundial da Saúde (OMS), embora muitos estudos sobre o tema tenham resultados questionáveis (LIMA 2016).

Portanto, é importante destacar que a idade materna avançada está fortemente associada ao risco de desenvolver neoplasia mamaria durante a gestação, visto que, após os 30 anos de idade é a patologia mais frequentemente diagnosticada, a uma larga distância das outras (COSTA et al., 2006). Fatores relacionados a hábitos de vida incluem a ingestão de bebida alcoólica, o sobrepeso e obesidade. O tabagismo é um fator que vem sendo estudado ao longo dos anos, com resultados controversos quanto ao aumento do risco de câncer de mama. Mas, na atualidade, existem algumas evidências de que ele pode sim aumentar as chances de surgimento deste tipo de tumor.

Mulheres com histórico de casos de câncer de mama em familiares consanguíneos, sobretudo em idade jovem, de câncer de ovário ou de câncer de mama em homem, podem ter predisposição genética para desenvolverem a doença. Portanto, apresentam um risco eminente, visto que mulheres com 30 anos de idade ou mais que apresentam este padrão, tendem a ter um risco três vezes maior do que 
as outras que não possuem histórico de parentes com câncer de mama (INCA, 2017).

\subsection{Mecanismos de rastreamento e Prevenção}

Segundo Rezende et al., (2005), a prevenção primária está relacionada com os hábitos de vida da mulher, como sua orientação dietética saudável, combate à obesidade, redução e até mesmo eliminação do uso do tabaco e do álcool, e principalmente uso criterioso de hormônios, em especial os anticoncepcionais.

Todavia, INCA (2017) enfatiza que as medidas preventivas do câncer de mama gestacional não são muito eminentes, pois são inúmeros fatores que se relacionam ao surgimento da doença, e ao fato de que a maioria destes não serem variáveis. Portanto, a prevenção para este tipo de enfermidade consiste no controle dos fatores de risco e no estímulo aos princípios protetores, especialmente aqueles considerados modificáveis. Pois se estima que por meio da alimentação saudável, e práticas de atividade física é possível reduzir em até $28 \%$ o risco de a mulher desenvolver a neoplasia mamaria. A amamentação também é considerada um fator protetor contra esta patologia.

Programas de rastreamento incentivando o exame de mamografia quando houver histórico da doença na família em qualquer idade. Como se sabe que o risco de ter câncer na gestação está ligado ao adiamento da gravidez, é aconselhável que as mulheres tenham filhos antes dos 32 anos de idade. $O$ auto exame da mama antes, durante e depois da gestação deve ser efetuado, pois sendo a doença diagnosticada o mais cedo possível facilita na escolha do método terapêutico a ser utilizado, a fim de assegurar a integridade da mãe e do feto (MOTTOLA Jr. et al., 2002).

\subsection{Métodos Terapêuticos}

Todavia, mesmo durante a gestação é possível tratar este tipo de tumor, pois o adiamento das intervenções pode ocasionar graves problemas de saúde para a mãe segundo (MONTEIRO et al., 2013). A proposta de tratamento do câncer de 
mama na mulher gestante é o mesmo da mulher não gestante, que consiste basicamente na prevenção de metástases sistêmicas e controle na região. No entanto, o modo como o tratamento é conduzido, é o que precisa ser avaliado cuidadosamente pela equipe de saúde responsável, em função dos eminentes efeitos adversos que podem vir a ocorrer ao feto.

As escolhas dos métodos terapêuticos que serão adotados na condução do tratamento irão depender de alguns fatores, como; tamanho do tumor, localização do mesmo, se a patologia está apenas em uma região ou se espalhou tempo gestacional, estado de saúde geral da paciente e principalmente as preferências pessoais dela. Devido ao fato de que, a gestante precisa conhecer todas as suas opções antes de tomar qualquer decisão, pois as escolhas de tratamento podem ser muito difíceis, principalmente porque podem gerar conflito entre o melhor tratamento para a mãe e o bem-estar do feto (SILVA, 2017). Portanto, esta paciente precisa ter um acompanhamento de uma equipe multidisciplinar, tendo ao seu lado um profissional obstetra, um (a) enfermeiro (a), um oncologista, um mastologista e um psicólogo, para dar um apoio emocional que esta mãe pode vir a precisar ao decorrer do tratamento.

Silva (2017) afirma que atualmente, as intervenções mais indicadas para o tratamento da neoplasia mamaria gestacional incluem: Cirurgia, Quimioterapia (QT) e após o parto a Radioterapia, hormonioterapia, e a terapia alvo, pois estes são mais propensos a causar malefícios ao feto em desenvolvimento dentro do ventre materno.

\subsection{Cirurgia}

Segundo Kettelhut et al. (2008), As diretrizes são as mesmas das pacientes não-grávidas dependente do estágio da doença e deve ser modificada de acordo com as necessidades materno-fetais. A cirurgia é usualmente o primeiro tratamento considerado. Pode ser realizada seguramente durante todos os trimestres de gestação com riscos mínimos ao feto em desenvolvimento. O cirurgião e a paciente podem optar por esperar até completar 12 semanas de gestação devido ao maior 
risco de abortamento neste período. Durante a cirurgia, a monitorização fetal deve ser considerada.

Nos artigos publicados, a maioria das pacientes gestantes com câncer de mama realizou mastectomia com linfadenectomia, provavelmente devido às considerações sobre o uso de radioterapia durante a gestação e ao estágio de apresentação mais avançado do tumor na época do diagnóstico. A mastectomia com linfadenectomia é o procedimento mais seguro nos tumores dos estádios I, II e III operáveis, devendo ser indicada em qualquer período. A cirurgiaconservadora em nódulos menores que $2 \mathrm{~cm}$ de diâmetro pode ser realizada principalmente nas mulheres que foram diagnosticadas no terceiro trimestre. (KETTELHUT, et al., 2008).

Já a Quimioterapia pode ser indicada no segundo ou terceiro trimestre, tratase da administração de drogas antineoplásicas, onde o fármaco é infundido em bólus em um acesso venoso periférico. O tratamento quimioterápico mais usado durante o segundo ou terceiro trimestre da gravidez consiste em 5-fluorouracil $(F)$, doxorrubicina $(A)$ ou epirrubicina $(E)$ e ciclofosfamida $(C)$ ou na combinação de doxorrubicina e ciclofosfamida (AC). Todos são considerados categoria $D$ na gestação. (ASHWORTH, 2016)

A radioterapia visa melhorar a sobre vida de mulheres mastectomizadas com alto risco, melhorando o controle a doença, porém esse método deve ser adiado de quatro a seis meses após o parto sem prejuízo de recorrência local, pois as radiações podem atrapalhar o desenvolvimento do feto. (MONTEIRO et al., 2013). No cenário atual, as evidências comprováveis quanto à segurança na utilização dos métodos terapêuticos mais indicados na condução do tratamento da neoplasia mamaria durante o período gestacional é muito limitada, principalmente quanto ao uso de fármacos quimioterápicos o que dificulta a tomada de decisão por parte da equipe multidisciplinar, uma vez que esta patologia não é muito comum, e tão pouco discutida e divulgada na sociedade. Porém, o fato das mulheres estarem preferindo adiar a primeira gravidez para a terceira e até mesmo quarta década de suas vidas, faz com que esta enfermidade não seja tão inesperada assim, e levanta a urgência de mais discussões a respeito da neoplasia mamaria na gestação. (SILVA, 2017). 
Maluf et al., (2005) aborda em seus estudos indagações relacionadas com os hábitos de vida da mulher moderna, que podem também influenciar no surgimento do câncer de mama gestacional. Dentre as nove pacientes $(60 \%)$ que tiveram o diagnóstico realizado durante o período gestacional, duas pacientes abortaram, sendo um espontâneo e outro por opção de tratamento conservador. As sete pacientes $(46,6 \%)$ restantes tiveram recém-nascidos $(R N)$ vivos sem malformações, sendo que dois $\mathrm{RN}$ apresentaram icterícia fisiológica. Uma paciente evoluiu para trabalho de parto prematuro, dando à luz na $32^{\text {a }}$ semana de gravidez, sendo que 0 RN não apresentou malformações (MOTTOLA JR et al., 2002).

Mottola JR et al (2002), ressalta que "o aborto não deve ser considerado uma medida integrante da terapêutica contra o câncer de mama associado à gravidez". Dado que, estudos retrospectivos já publicados não constataram aumento da sobrevida nas doentes submetidas à interrupção da gestação. Desta forma, a indicação de aborto terapêutico deve ser orientada para aquelas enfermas nas quais a patologia, consiste em danos fetais, desencadeados pela intervenção adjuvante, como a radioterapia e quimioterapia. Mas o fato de deliberar a prática do aborto nestas pacientes, precisa sempre ser uma escolha em conjunto de toda a equipe multidisciplinar, visando oferecer todo o apoio social e psicológico para a paciente e sua família.

Portanto, vale salientar a necessidade de orientar as pacientes que tiveram câncer de mama gestacional ou que já deram à luz e estão em tratamento adjuvante, a extrema importância da anticoncepção, que precisa ser executada utilizando-se dispositivo intra-uterino, métodos de barreira ou associação dos métodos não hormonais. Sendo assim, é estipulado, o exame clínico apropriado e exames de imagem, sendo a Ultrassonografia Transvaginal (USG) para mulheres com idade inferior a 35 anos e a mamografia para mulheres com idade acima de 35 anos na fase pré-concepcional. Já nas primeiras consultas pré-natais, é aconselhável a realização de um exame clinico mais rigoroso, pois neste período a probabilidade de encontrar uma neoplasia mamaria é maior do que nos demais trimestres da gravidez, uma vez que ocorre aumento da hipertrofia e do ingurgitamento mamário. Ao aparecimento de qualquer nódulo percebido durante a 
gestação, a investigação clínica, radiológica e cito-histológica não pode ser adiada (MOTTOLA JR et al., 2002).

\section{Metodologia}

\subsection{Tipo de Estudo}

Este estudo é de caráter bibliográfico com o intuito de agregar conhecimento acerca do tema em debate, destacando os métodos terapêuticos indicados na condução do tratamento do câncer de mama gestacional.

\subsection{Fontes pesquisadas}

As buscas de conteúdos relativos ao tema proposto foram direcionadas por sites de pesquisas banco de dados da Biblioteca Virtual em Saúde (BVS) utilizando, como fontes, a Base de Dados da Enfermagem (BDENF) e a Scientific Eletronic Libray Online (SCIELO), Google Acadêmico, Instituto Nacional do Câncer (INCA). A consulta atendeu ao critério da presença dos Descritores: "Neoplasia da Mama", "Gestação", "Complicações neoplásicas na Gravidez", "Tratamento" e "Fatores de riscos".

\subsection{Critério de inclusão e exclusão}

Este estudo baseia-se na pesquisa de artigos publicados em periódicos científicos nacionais. Foram utilizados os seguintes critérios.

\section{Critérios de inclusão:}

- Disponibilidade do artigo na íntegra;

- Publicados em português;

- Publicados no período de 2000 a 2020;

- Artigos disponíveis gratuitamente on-line;

- Seleção do título que contenha referência aos descritores;

- Leitura classificatória do resumo e leitura do texto na íntegra (também classificatória);

\section{Critérios de Exclusão:}


- Artigos duplicados;

- Artigos que não tenham sido publicados nos últimos 10 anos;

\subsection{Coleta de dados}

Utilizando os descritores citados, encontrou-se um total de 500 artigos, foram selecionados a partir da leitura de Título e Resumo. Destes, foram escolhidos para ser lidos na íntegra e verificar sua relevância com o tema, sendo selecionados 20 artigos para fazer parte desta revisão integrativa.

A coleta de dados foi instrumentalizada através de um quadro bibliográficos quadro (APÊNDICE A), foram evidenciadas as informações referentes a cada autor como: Ano, Autores, Fonte e Título. Após a coleta desses dados, as autoras obtiveram informações que subsidiaram a criação dos resultados da pesquisa, a qual foi realizada através do debate dos autores por meio dos quadros fundamentados pelos resultados encontrado

\section{Resultados e Discussão}

Esta pesquisa se apresenta como um trabalho bibliográfico, exploratório e descritivo por apresentar embasamento bibliográfico que expõe dados coletados por pesquisadores e conceitos que estimulam a compreensão do tema. Selecionou-se este tipo de abordagem por se entender que esta seja a melhor forma de compreender a complexidade deste tema e suas implicações na vida das pacientes.

O levantamento das fontes elencadas para embasar este estudo teve inicio em agosto de 2019 e a sistematização se estendeu por todo primeiro semestre de 2020. A edificação da pesquisa foi feita a partir da apreciação dos objetivos deste trabalho para que ao final do processo fosse possível atingi-los. 
No decorrer da sistematização das ideias foi possível compreender conforme a caracterização de Thuller et al., (2003), o câncer de mama é uma doença causada pela multiplicação desordenada de células da mama e há vários tipos dessa neoplasia da mama e devido a este fator ele se torna difícil de ser tratado. Além disto, esta patologia pode ser mais difícil de ser diagnosticada quando a mulher está grávida porque as alterações hormonais durante a gravidez fazem com que as mamas se tornem maiores, granuladas e/ou sensíveis, o que dificulta o diagnóstico.

Constatou-se segundo Cipriano et al., (2015) que no Brasil, o câncer de mama é a neoplasia maligna que mais ataca as mulheres e é o segundo mais frequente, ficando atrás somente do de pulmão. Esta neoplasia mamária perde apenas para as doenças cardiovasculares como a doença que mais mata e 0 diagnóstico tardio é responsável por grande parte dos óbitos.

Outro fator recorrente apontado por Grendys Jr. (2010), apud, COSTA et al., (2006) refere-se ao fato de que os países subdesenvolvidos serão responsáveis por 80\% dos mais de 20 milhões de casos novos de câncer estimados para 2025, o que pode ser associado à falta de informação suficiente e prevenção necessária para o caso. Outro fator relevante apontado por estudo é o de que, a maioria dos estudos realizados apontarem que a gravidez não é um fator de prognóstico deficiente, mas durante a gravidez, o processo, pode se desenvolver de forma mais agressiva, também devido ao atraso no diagnóstico e tratamento. Desse modo, quanto mais cedo for descoberta mais as chances de cura da paciente aumentam, haja vista que se pode optar por métodos terapêuticos mais eficazes para o sucesso do tratamento. (MOTTOLA JR et al., 2002).

Portanto, vale salientar a necessidade de orientar as pacientes que tiveram câncer de mama gestacional ou que já deram à luz e estão em tratamento adjuvante, a extrema importância da anticoncepção, que precisa ser executada utilizando-se dispositivo intra-uterino, métodos de barreira ou associação dos métodos não hormonais. Sendo assim, é estipulado, o exame clínico apropriado e exames de imagem, sendo a Ultrassonografia Transvaginal (USG) para mulheres com idade inferior a 35 anos e a mamografia para mulheres com idade acima de 35 anos na fase pré-concepcional. Já nas primeiras consultas pré-natais, é aconselhável a realização de um exame clinico mais rigoroso, pois neste período a 
probabilidade de encontrar uma neoplasia mamaria é maior do que nos demais trimestres da gravidez, uma vez que ocorre aumento da hipertrofia e do ingurgitamento mamário. Ao aparecimento de qualquer nódulo percebido durante a gestação, a investigação clínica, radiológica e cito-histológica não pode ser adiada (MOTTOLA JR et al., 2002).

Este estudo aponta ainda como fator pertinente ao crescimento deste tipo de neoplasia a opção das mulheres por adiar cada vez mais a gestação e como consequência disso serem mais acometidas por este tipo de doença que geralmente acomete mulheres em idade adulta. Desse modo, para um diagnóstico precedente e maior chance de um tratamento eficiente, a ultrassonografia das mamas e a mamografia são indicadas sempre que houver lesão suspeita na mama da gestante, associado aos sinais e sintomas anteriormente citados. (INCA, 2017). Lembrando que conforme Mottola Jr, et al (2002) em qualquer tratamento, a descoberta da patologia precocemente leva a maiores chances de cura, contudo no caso da gestante tem um tratamento diferenciado deve ser ministrado, pelo fato de apresentar alterações hormonais provenientes da própria gestação.

Observou-se que os fatores de risco apontados por Lima (2016) para esta neoplasia ainda não são muito definidos e estudiosos apontam como possíveis causas os problemas endócrinos, idade, fatores relacionados a hábitos de vida e fatores genético-hereditários. Mas a OMS destaca como fator de máxima relevância, a idade materna avançada, salvo que, após os 30 anos de idade, é a patologia comumente diagnosticada, em larga escala se comparada à outras.

Como mecanismos de rastreamento e prevenção foram apontados por Rezende et al. (2005), fatores relacionados com os hábitos de vida da mulher, sua orientação dietética no combate à obesidade, redução e/ou eliminação do uso do tabaco e álcool e, sobretudo, uso razoável de hormônios, particularmente os anticoncepcionais. Entretanto o INCA (2017) estima que por meio da alimentação saudável e práticas de atividade física é possível reduzir em até $28 \%$ o risco de a mulher desenvolver a neoplasia mamária. Outro fator apontado como protetivo é a amamentação. O rastreamento incentivando o exame de mamografia quando houver histórico da doença na família em qualquer idade é também considerado um fator de prevenção. 
Conforme Monteiro et al., (2013) a proposta de tratamento do câncer de mama na mulher gestante o mesmo tratamento da mulher não gestante, que consiste basicamente na prevenção de metástases sistêmicas e controle na região. Contudo devido aos efeitos adversos que podem vir a ocorrer ao feto, o acompanhamento da mulher gestante deve ser mais cauteloso.

Em relação à cirurgia constatou-se observando as premissas de Kettelhut et al., (2008), que ela é indicada para as mulheres que se encontram nesta condição e são adotados os mesmos procedimentos das pacientes não-grávidas, porém conforme o estágio da doença a técnica deve ser modificada de acordo com as necessidades materno-fetais. A cirurgia é usualmente o primeiro tratamento considerado. Pode ser realizada seguramente durante todos os trimestres de gestação com riscos mínimos ao feto em desenvolvimento, porém o cirurgião e a paciente podem optar por esperar até completar 12 semanas de gestação devido ao maior risco de abortamento neste período. Tanto a quimioterapia quanto radioterapia podem ser indicadas após o processo mastectômico, contudo o profissional deve avaliar os riscos para o desenvolvimento do feto.

É importante ressaltar que conforme Mottola JR et al., (2002), experiências realizadas em nove pacientes que tiveram o diagnóstico realizado durante o período gestacional, duas pacientes abortaram, sendo um espontâneo e outro por opção de tratamento conservador. As sete pacientes restantes tiveram recém-nascidos vivos sem malformações, sendo que dois deles apresentaram icterícia fisiológica. Uma paciente evoluiu para trabalho de parto prematuro, dando à luz na $32^{\mathrm{a}}$ semana de gravidez, sendo que o bebê não apresentou malformações. Assim, diante de tais informações, cabe pontuar que o aborto não deve ser considerado uma medida complementar do tratamento contra o câncer de mama gestacional a não ser em casos onde o feto apresentar má formação devido à complementação terapêutica. Contudo ao indicar um aborto se faz necessário cuidados sociais e psicológicos com a paciente e sua família para que problemas não surjam em decorrência desta decisão.

\section{Considerações Finais}


Ao iniciar este trabalho elencaram-se os seguintes objetivos:

Investigar os métodos terapêuticos indicados na condução do tratamento do câncer de mama gestacional.

Listar os fatores de risco que podem desencadear o câncer de mama durante a gestação; Conceituar a patologia; Destacar métodos preventivos existentes.

A problematização levantada foi: "Quais são os métodos terapêuticos indicados na condução do tratamento do câncer de mama gestacional?"

A seguir expuseram-se os principais aspectos da patologia, também denominada neoplasia e reverenciou-se uma gama de teóricos para alicerçarem o estudo.

Posteriormente foi feita a análise dos dados apresentados pelo referidos autores para maior compreensão dos dados por eles mencionados.

Por fim, realizou-se a tarefa de balizar estas considerações finais acerca de um tema que se considera ainda carente de estudos e bibliografias que apresentem maiores suportes a porvindouras pesquisas. Entendeu-se no decorrer deste estudo que a experiência tanto teórica quanto a prática nos ambientes hospitalares são fatores que muito contribuem para o para a compreensão da realidade das pacientes diagnosticadas com esta neoplasia.

\section{Referências}

ARAÚJO, A. M,; CENTENO, M. Cancro da mama associado à gravidez. Faculdade de Medicina de Lisboa. p.44-52. Lisboa: 2010.

ASHWORTH, J.; LOPES, E.O. Cancro da mama associado à gravidez- efeito da quimioterapia no feto. 2016. Tese (Mestrado Integrado em Medicina Artigo de Revisão). Instituto de Ciências Biomédicas Abel Salazar. Universidade do Porto. Porto. Portugal. Disponível em: https://repositorioaberto.up.pt/bitstream/10216/90024/2/168705.pdf

AVELAR, P.M; SILVA, M.L; PASSOS, S.X. O Papel da Enfermagem no Câncer de Mama. Área temática: Saúde da Mulher. Goiânia-GO. p.10.18, Novembro: 2014.

BASTOS, F. A. C.; MONTEIRO, I. M. Câncer de mama e gestação: Relato de caso. Trabalho realizado na Fundação Santa Casa de Misericórdia do Pará, s/d. 
BRASIL, M. S. Secretaria de Atenção à Saúde. Departamento de Ações Programáticas Estratégicas. Gestação de alto risco: manual técnico / Ministério da Saúde, Secretaria de Atenção à Saúde, Departamento de Ações Programáticas Estratégicas. - 5. ed. - Brasília : Editora do Ministério da Saúde: 2010. 302 p. - (Série A. Normas e Manuais Técnicos) ISBN 978-85-334-1767-0 1. Gestação de alto risco. 2. Gestante de risco. 3. Obstetrícia. I. Título. II. Série.

BRITO, E.A.S., et al. Diagnóstico de Câncer durante a Gestação: Uma Revisão Integrativa. Id on Line Rev. Mult. Psic. v.14, n. 49 p. 150-161, Fevereiro/2020. Edição eletrônica disponível em http://idonline.emnuvens.com.br/id Acesso em 20/05/2020.

CARNEIRO, E.C.S.P., et al. A percepção da mulher com câncer mamário em relação ao impacto nos filhos. Revista Cubana de Enfermería. 2020. Edição eletrônica disponível em https://creativecommons.org/licenses/by-nc/4.0/deed.es_ES Acesso em 20/04/2020.

CIPRIANO, P.; SC, C. O, . Gestação e câncer de mama: proposta de guia de orientações. Fisioterapia Brasil. Ano 2015. vol. 16. Número 3.

COSTA, C. L.; R.; LOURES, L. F.; ARAÚJO, D.A.C.; SOUZA, L.C.. Câncer de mama durante a gestação: revisão bibliográfica. Juiz de Fora,v.32,n.4,p.109114,out./dez.2006.

https://hurevista.ufjf.emnuvens.com.br/hurevista/article/viewFile/26/21

DIAS, M.N.S.. O enfrentamento do problema do câncer de mama na estratégia de saúde da família. Trabalho de conclusão de curso apresentado ao curso Especialização em Atenção Básica em Saúde da Família. UFMG. Conselheiro Lafaeite: 2009

FERNANDES, A.F.C; SANTOS, M.C.L; SILVA, T.B.C; Galvão C.M. O prognóstico de câncer de mama na gravidez: evidências para o cuidado de enfermagem. Rev. Latino-Am. Enfermagem19(6):[10 telas] nov.-dez. 2011.www.eerp.usp.br/rlae

FERREIRA. L.R.G; SPAUTZ, C.C. Câncer de mama associado à gestação. Femina. Julho|agosto2014.vol 42.no 4.

INSTITUTO NACIONAL DO CÂNCER (INCA). Falando sobre câncer de mama. Disponível em: <http://www.inca.org.br>. Acesso em: 01/01/2020.

KETTELHUT, J.C.; MODENA, M.A.B.. Câncer de mama e gestação. Rev. Fac. Ciênc. Méd. Sorocaba, v. 10, n. 4, p. 1 - 4, 2008.

LIMA, L.V.G.C.. Cancro da mama associado à gravidez. 2016. Tese ( Mestrado Integrado em Medicina Artigo de Revisão ). Instituto de Ciências Biomédicas Abel Salazar. Universidade do Porto. Porto. Portugal. Disponível em: https://repositorioaberto.up.pt/bitstream/10216/88969/2/169901.pdf 
LUCARELLI, A.P.; MARTINS, M. M.. Câncer de mama e gestação. Revista FEMINA. Julho/Agosto 2012. vol.40.n 4.

MALUF, M. F. M.; JO, M. L; BARROS, A. C. S. D. O impacto psicológico do câncer de mama. Rev. bras. cancerol. v.51, n.2, p. 149-154, 2005.

MONTEIRO, M.L.D; TRAJANO, B.J.A, et al. Câncer de mama na gravidez e quimioterapia: Revisão sistemática. rev.assocmed bras.2013;59(2):174-18.

MORENO, M. L. O papel do enfermeiro na abordagem do câncer de mama na estratégia de saúde da família. Trabalho de conclusão de curso apresentado ao curso Especialização em Atenção Básica em Saúde da Família. UFMG. Uberaba: 2010.

MOTTOLA, JR, J. et al. Câncer de mama associado à gravidez: um estudo caso/controle. Rev. bras. ginecol. obstet., v.24, n.9, p.: 585-591, 2002.

PRADO, N., et al. Gestante com diagnóstico de câncer de mama: prevenção, diagnóstico e assistência. Braz. J. Hea. Rev., Curitiba, v. 3, n. 1, p. 1109-1131 jan./fev. 2020.

REZENDE, W. W. et al. Câncer de mama associado à gravidez: revisão de literatura. Femina, v.33, n.6, p. 435-442, 2005.

SCHÜNEMANN, J. E., et al. Radioterapia e quimioterapia no tratamento do câncer durante a gestação - revisão de literatura. Revista Brasileira de Cancerologia 2007; 53(1): 41-46.

SILVA, I. Artigo de revisão: o câncer de mama na gestação e a condução do tratamento. Ponto dos concursos. BA, 12 de abril, 2017. Disponível em: https://www.pontodosconcursos.com.br/Artigo/VisualizarArquivo?id=257. Acesso em: 01/01/2020.

SPAUTZ, C.C.; FERREIRA, L.R.G. Câncer de mama associado à gestação. Estudo realizado no Departamento de Tocoginecologia do Hospital de Clínicas da Universidade Federal do Paraná (HC/UFPR). vol 42.nํ⒋ Curitiba (PR): Brasil. 2014.

VIEIRA, R.I.S.; GOMES, R.; TRAJANO, A.I.B. Câncer de mama e gravidez subsequente: um olhar sociocultural. Rev. bras. cancerol., v. 51, n. 2, p. 102-110, 2005. 